\title{
Study on Effectiveness of Static Stretching and Massage on Hamstring Flexibility in Normal Adults
}

\author{
Mamta Boora ${ }^{1}$, Sujata Sharma ${ }^{2}$ \\ ${ }^{1,2}$ Assistant Professor, Department of Physiotherapy, GJUS\&T, Hisar, Haryana, India.
}

\begin{abstract}
Introduction: Muscle tightness is the most common disorder in normal healthy individuals and insufficient range of motion caused by poor muscle flexibility may be a cause of muscle strain and increased risk of injury. Hamstring muscles are reported to be the most commonly injured multijoint muscle group in the body because strains are particularly common in multijoint muscles which have a greater functional excursion and tend to have a higher concentration of fast twitch muscle fibers.Tightness of hamstring muscle has shown to predispose a person to several musculoskeletal overuse injuries and significantly affects person level of function. To enhance from the health perspective it is typically necessary to engage muscle in systematic stretching and massage over a period of time.
\end{abstract}

Purpose: To compare the effectiveness static stretching, musculotendinous massage and combination therapy on hamstring flexibility. Presently no documentation suggesting which one is better and whether any significant difference between stretching, massage and combination therapy.

Methodology: Forty five subjects were included in the study. The subjects were randomly divided into three groups by using simple random sampling method. Group A received static stretching, Group B received massage and Group C received combination of both.

Result: Related t-test was used and the value of $t$ in stretching, massage and combined therapy group were 5.917, 6.948 and10.191 which were statically significant at the level of $p<0.05$. ANOVA indicated a significant interaction between the three groups and test in degrees of knee extension.

Conclusion: This study demonstrated that that static stretching and massage both are equally effective to increase the hamstring flexibility but combined therapy exhibit significant improvement as compare to static stretching and massage group.

\section{Introduction}

Muscle is a prime mover and stabilizer of body that contains muscle spindle as its functional unit and golgi tendon organs in determining the length and function of muscular components. The length of muscle tissue is thought to play an important role in the effectiveness and efficacy of human movement which is affected by many factors like, age, gender, genetic factors, sedentary lifestyle and immobilization etc. ${ }^{1}$

Muscular flexibility is defined as the ability of a muscle to lengthen, allowing one joint (or more than one joint in a series) to move through a range of motion without any discomfort and a loss of muscle flexibility as decrease the ability of the muscle to deform resulting in decreased ROM about a joint. Muscular flexibility is an important aspect of normal human function and limited flexibility has been shown to predispose a person to several musculoskeletal overuse injuries and significantly affect a person's level of function. ${ }^{2}$

Hamstrings are the long and powerful group of muscles that span the back of the thigh.Hamstring tightness is the inability to stretch the muscle through full range of amplitude and tightness of this muscle can play a role in sport related injuries, lumbar spine disorder and general low back pain. The hamstring muscle also reported to be the most commonly injured multijoint muscle group in the body because strains are particularly common in multijoint muscles which have a greater functional excursion and tend to have a higher concentration of fast-twitch muscle fibers.

Muscle tightness is the most common disorder in normal healthy individuals and this insufficient range of motion (ROM) caused by poor muscle flexibility may be a cause of muscle strain and increased risk of injury so to enhance ROM from a health perspective it is typically necessary to engage muscle in systematic stretching over a period of time. ${ }^{1}$

\section{Methodology}

Study design used was experimental. Method of sampling was Subjects who have hamstring tightness were selected through passive knee extension test for the study. The whole sample composed of 45 subjects. The subjects were randomly divided into three groups by using Simple

Random Sampling method. Each group consist of 15 subjects. In a 1 week treatment program each group received the therapy for 1 time per day. Group A consist of 15 subjects who received static stretching, group B 
consist of 15 subjects who received massage and group $\mathrm{C}$ also consist of 15 subjects who received combination of both two . All the subjects were informed that they are under the experiment and a prior consent of subject was sought before assessment. Inclusion Criteria of this study was, Subjects with hamstring tightness, only young adults were included in the study without any significant history of pathology of the hip, knee, thigh or low back. Exclusion Criteria of this study was Individuals with back pain, visceral pain, soft tissue injury, fracture and recently operated individuals. Instrumentation includes a wooden table, a marker pen and stop watch, metallic conventional universal goniometer and an assessment format.

\section{Procedure}

To participate in the study subjects must have exhibited tight hamstring muscle defined as having greater than $20^{\circ}$ loss of knee extension which was measured with the help of passive knee extension test . In addition, subjects who were not involved in any exercise activity at the start of the study had to agree to avoid lower-extremity exercise and activities other than those prescribed by the research protocol. Subjects who were involved in exercise activity at the start of the study agreed not to increase the intensity or frequency of the activity during the 1 weeks of training.

\section{Passive knee extension test procedure:}

All subjects who met the criteria for inclusion in the study and signed the informed consent were measured for flexibility of the hamstring muscles prior to assignment to groups. Each subject was positioned supine with the tested hip and knee flexed to $90^{\circ}$ and apposite lower extremity extended.

The lateral malleolus, lateral epicondyle of the femur and greater trochanter of the right lower extremity were then marked with a marker pen for later goniometric measurement. Ninety degrees of hip flexion was maintained by assistant, while the tibia of the knee was passively moved to the terminal position of knee extension by the researcher The terminal position of knee extension was defined as the point at which the subject complained of a feeling of discomfort or tightness in the hamstring muscles or the experimenter perceived resistance to stretch. Once the terminal position of knee extension was reached, the examiner measured the amount of knee extension with the goniometer. $0^{0}$ of knee extension were considered full hamstring muscle flexibility. Both premeasurements and postmeasurements were taken by this procedure.

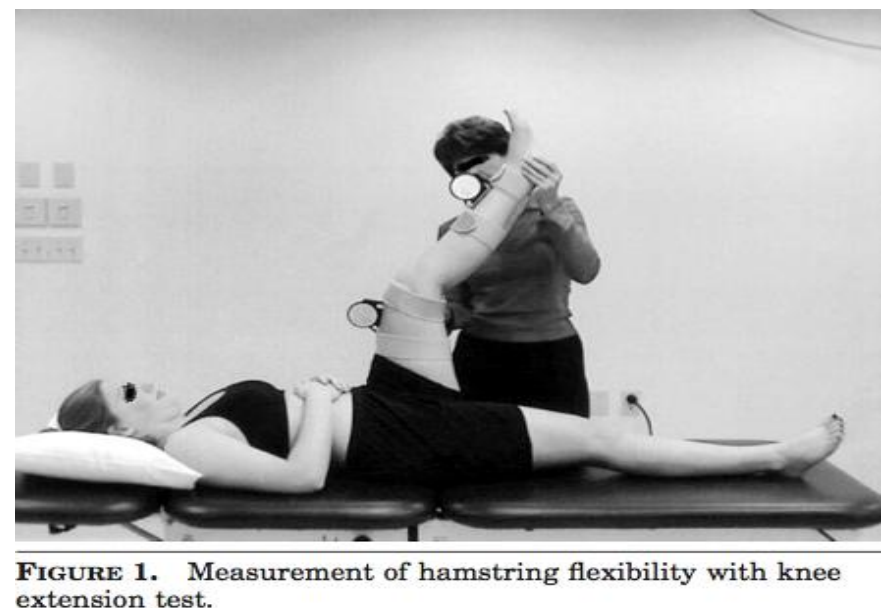

\section{Static stretching procedure}

Following pretesting, the subjects were randomly assigned into three groups. Stretching of hamstring muscle will performed by straight leg raising technique. In Group A ,6 men, 9 women was assigned to participate in passive static stretching sustained for 30 seconds, in Group B, $7 \mathrm{men}, 8$ women was assigned to participate in massage at musculotendinous junction and in Group C ,6 men, 9 women was assigned to receive combination of both static stretching and massage.

Subjects in Groups A to $\mathrm{C}$ treated for one time a day for 1 week. Each subject knee was passively moved to extension with the ankle 90 degrees and the subjects were instructed to keep their back as flat as possible. Subjects received 3 stretches for their designated time period (30 second) with 10 second rest in between for 1 week. During 10 second rest all subjects will asked to move the lower extremity back and forth from knee to chest in order to prevent thixotrophic stiffening.

\section{Massage Procedure}

Friction massage was applied at the musculotendinous junction of the distal portion of the hamstrings characterized as an accurately delivered penetrating pressure applied through the fingertips. This pressure was 
applied in small circular motions with deep pressure by the same investigator for each subject. The subject was in supine position and his knee was extended. The investigator grasped the subject's thigh with both hands (thumbs on the quadriceps) and applied friction pressure in circular motions with the fingers at the musculotendinous junction of the hamstrings for 3 times per day for 30 seconds for 1

\section{Data Analysis}

Statistical analysis was performed using the software package SPSS version 17 . The mean values of prestretching - poststretching and premassage - postmassage were calculated for affected leg. The mean values of the improvement in passive knee extension test was made by static stretching , massage and combined therapy .The amount of standard deviation in prestretching- poststretching and premassage-postmassage was also calculated. The related t- test was performed on the collected data and ANOVA was calculated.

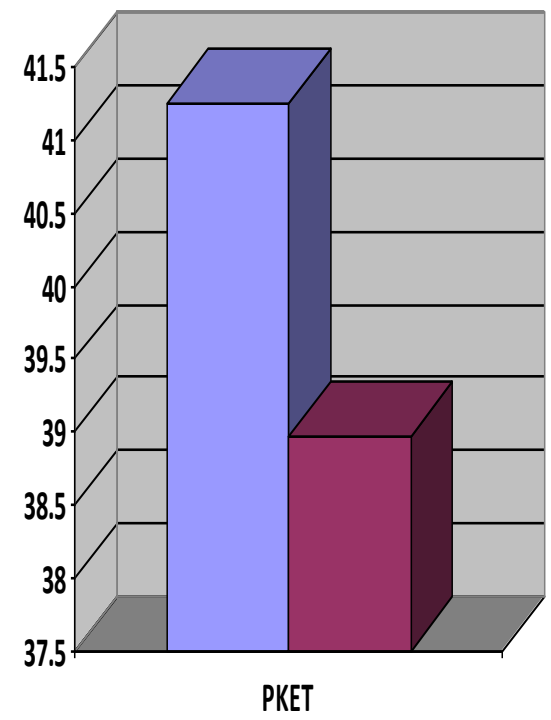

In Group -A, the PKET mean, Standard deviation and $t$ value for pre And post readings were $39.509 \pm 6.001$, $38.970 \pm 3.537$ and $t$-value was 5.917

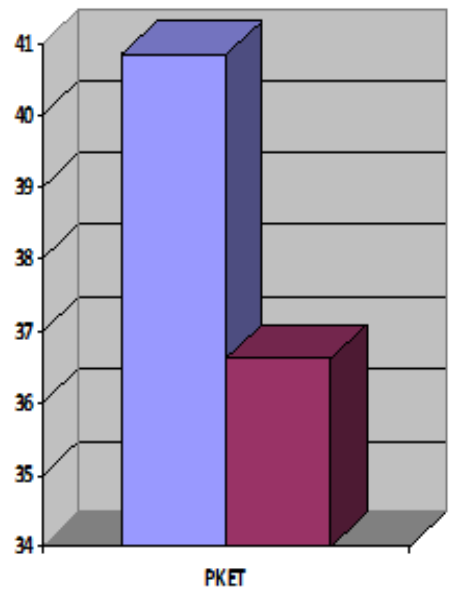

In Group -C, the PKET mean, Standard deviation and $t$ value For pre and post readings were $40.841 \pm 6.394,36.619 \pm 5.809$

And t-value was 10.191

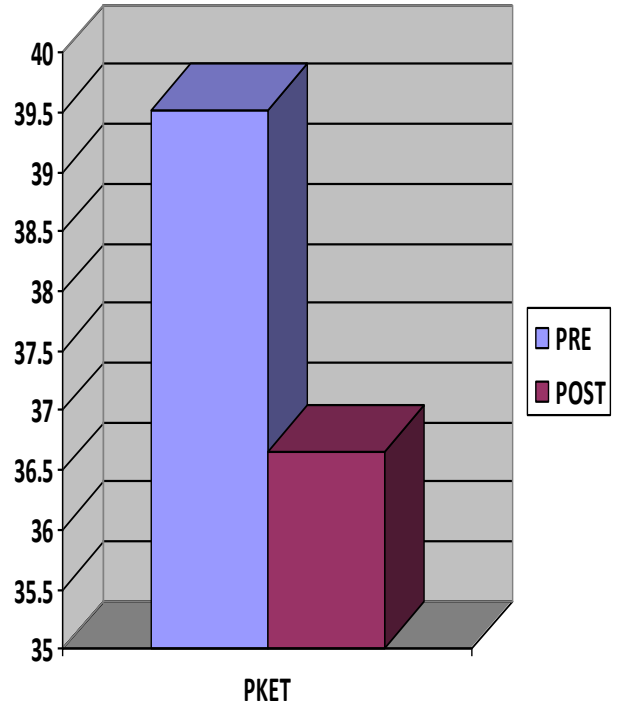

In Group -B, the PKET mean, Standard deviation and $t$ value for pre and post readings were $41.242 \pm 4.568$, $36.646 \pm 4.963$ and $t$-value was 6.948

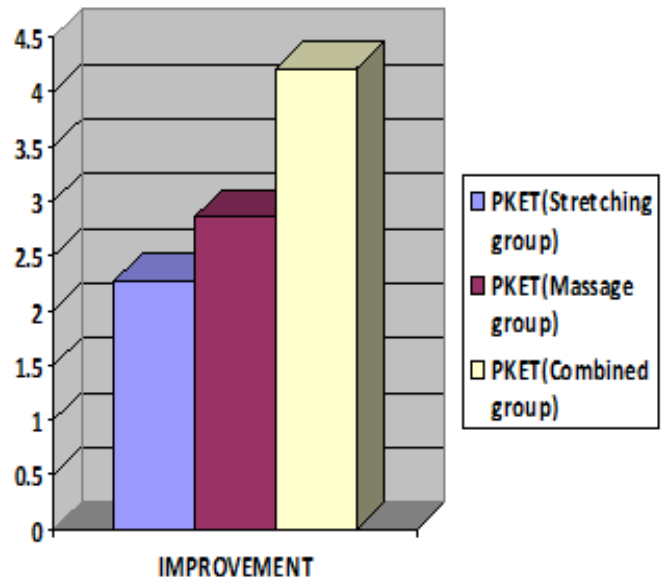

Improvement in PKET scores of stretching massage and combined therapy were seen and the improved mean and standard deviation values were $2.27 \pm 1.48,2.86 \pm 1.59$ and $4.22 \pm 1.60$ 


\section{Result}

After analysing data, significant difference was found between pre-stretching and post-stretching measurements of three different groups. The value of $t$ calculated through related $t$-test. Through related $t$-test, the value of $t$ in stretching group, massage group and combined therapy group were 5.917,6.948 and 10.191 which were statically significant at the level of $\mathrm{p}<0.05$. So the experimental hypothesis i.e. static stretching, massage and combined therapy were effective in improving hamstring flexibility in normal subjects.

The ANOVA indicated a significant interaction between the groups (stretching, massage and combined therapy group) and test (pre-test and post-test) in degrees of knee extension. Further evaluation of the data indicated that the change in degrees of knee extension for the subjects in both groups A and B, who were given static stretching and massage respectively have no significant difference from each other but group C who was given combined therapy resulted in higher degrees of gain in knee extension range which was considered as significantly different from group A and group B . So, the experimental hypothesis i.e. stretching and massage both were effective to increase knee extension ROM and combined therapy produce an additional effect was accepted and null hypothesis was rejected

\section{Discussion}

Muscle tightness is one of the limiting factors for restricted range of motion and reduced flexibility of joint. Hamstring muscles are more prone for tightness causes musculoskeletal problems. This study focused on checking effects of static stretching and massage in increasing ROM and flexibility of subjects with hamstring tightness. So according to our study both static stretching and massage techniques were effective at increasing hamstring flexibility.

The results of this investigation support the findings of Bandy and Irion and Bandy and colleagues, who concluded one 30-second static stretch is an effective dose for increasing hamstring flexibility. However, Bandy and colleagues used a frequency of 5 days per week for 6 weeks. Short-duration massage at the musculotendinous junction without additional stretching can provide ROM improvements similar to other stretching techniques.

The most important finding of the current study was the significant increases in knee extension ROM with only 30 seconds musculotendinous massage of hamstring 3 times in a day for one week. Previous studies have reported conflicting results with a single bout of 15 minutes of massage eliciting no significant changes in ROM with adolescent soccer players and active adults contrasting with 3 other studies reporting increased hamstrings flexibility with 8 minutes and 3 minutes of massage.

The present study indicates that a brief duration massage at the musculotendinous junction can provide an increase in hamstrings flexibility (knee extension ROM) that is comparable to other common methods of stretch .Increases in muscle-tendon compliance may be achieved with massage by mobilizing and elongating shortened or adhered connective tissue. Another possibility is that the massage caused increases in skin and muscle temperatures leading to augmented blood flow to the area. Although Drust et al. reported increased skin and muscle temperatures with massage; their massage duration was a minimum of 5 minutes compared with a maximum of 30 seconds in the present study.

\section{Conclusion And Future Study}

This study demonstrated that static stretching and massage both are equally effective to increase the hamstring flexibility. In addition, the combined therapy exhibit significant difference from static stretching and massage. Enhanced understanding of the effect of stretching and massage on the hamstring muscles as a result of the findings of our study will hopefully enable clinicians to provide more effective and scientifically based treatment when incorporating stretching and massage activities into rehabilitation programs. Limitations of study were small sample size. The outcome measures for muscle length was not used and analysed. Only subjective range of motion was measured by goniometer and only knee extension range of motion was measured and analysed,duration of the study was less for the follow - up of the outcome.It should also be noted that this study only investigated the effects only on asymptomatic individuals so results of the studies reported here may not apply directly to patient populations.Hamstring muscles were the only muscles tested, it would be inappropriate to generalize the results of this investigation to other muscles or muscle groups. Scopes of further study are Hip movement can also included with knee movement for better analysis. Duration of stretching and massage can be increased.These therapies can be studied for a longer duration of time for long term relief of condition

\section{References}

[1] D Scott Davis, Paul E.Ashby, Kristi L: The effectiveness of 3 stretching techniques on hamstring flexibility using consistent stretching parameters. Journal of Strength and Conditioning Research, 2005, 19(1), 27-32.

[2] Bandy W.D, Irion JM Briggler .M: The effect of time and frequency of static stretching on flexibility of hamstring muscles. Journal of Physical therapy. 1997; 77: 1090-1096. 
[3] Bandy WD, Irion JM.: The effect of time on static stretch on the flexibility of the hamstring muscle, physical therapy: 1994-74: 845-852.

[4] Wilkinson A: A review of the literature on muscle stretching. Australian Journal of physiotherapy 2005 : $38283-287$.

[5] Gajdosik RL: Effects of static stretching on the maximal length and resistance to passive stretch of short hamstring muscle. Journal ortho sports physical therapy 1991:14: 250-255.

[6] Halbertsma JP, Goeken LN: Stretching exercises effect on passive extensibility and stiffness in short hamstring of healthy subjects. Archives Physical Med Rehabilitation 1994: 75: 976 - 981.

[7] Halbertsma JP, Bolnuis A1: Sports stretching effect on passive muscle stiffness of short hamstring. Archives physical Medicine rehabilitation 1996: 77: 688-692

[8] Magnusson S.P: Passive properties of the hamstring muscle during stretch maneuver, A review. Journal of Medicine \& Science in Sports, 1998 8, 65-77.

[9] Taylor D, Dalton D: Visco elastic, properties of muscle tendon units; The biomechanical effects of stretching .American Journal of Sports Medicines .2001, 300-309.

[10] James et.al; Static stretching of knee extension range of motions: Journal of Orthopaedic and sport physical therapy, 2003: 35:12.

[11] Bandy WD, Irion JM, Briagler M. The effect of static stretch and dynamic range of motion training on the flexibility of the hamstring muscles:Journal of Orthopaedics sports physical Therapy, 1998; 27: 295-300.

[12] J Brent Feland, Jw Myrer, SS Schulthies, Gary W Meason: The effect of duration of stretching of the hamstring muscle group for increasing range of motion in people aged 65 years or older : Journal of Physical Therapy, 2001;81;110-117.

[13] Laura C. Decoster, Joshua Cleland, Carolann Altieri: The Effects of Hamstring Stretching on range of motion: A Systematic Literature Review: Journal of Physical Therapy, 2005; 35:377-387.

[14] Gogio P, Brate JH, Reliability and validity of goniometric measurements at the knee. Physical therapy 1987; 67: 192-195.

[15] Rushton A, Spencer S. The effect of soft tissue mobilisation techniques on flexibility and passive resistance in the hamstring muscle-tendon unit: A pilot investigation, 2005; 35:377-387.

[16] Stacey Y. Huang, Mario di santo,2 katie p. wadden Short-duration massage at the hamstrings musculotendinous junction induces greater range of motion:Department of Human Performanee and Exercise Science,2010,67:244:260.

[17] Moller M, Ekstrand J, Oberg B, Gillquist J: Duration of stretching effect on range of motion in lower extremities. Archie Phys Med Rehabilitation. 1985; 66:171-173.

[18] Chan SP, Hong Y, Robinson PD: Flexibility and passive resistance of the hamstrings of young adults using two different static stretching protocols. Scand J Med SciSports. 2001; 11:81-86.

[19] Hartig DE, Henderson JM.: Increasing hamstring flexibility decreases lower extremity overuse injuries in military basic trainees. Am J Sports Med. 1999;27:173-176

[20] Hopper, D, Deacon, S, Das, S, Jain, A, Riddel, D, Hall: Dynamic soft tissue mobilisation increases hamstring flexibility in healthy male subjects. Br J Sports Med, 2005, 39: 594-598.

[21] Barlow, A, Clarke, R, Johnson, N, Seabourne, B, Thomas, D, andGal, J. Effect of massage of the hamstring muscle group on performance of the sit and reach test .Br J Sports Med,2004, 38: 349-351, 